\title{
Protective and therapeutic effects of exercise on stress-induced memory impairment
}

\author{
Paul D. Loprinzi ${ }^{1} \cdot$ Emily Frith $^{1}$
}

Received: 3 May 2018 / Accepted: 5 September 2018 / Published online: 10 September 2018

(c) The Physiological Society of Japan and Springer Japan KK, part of Springer Nature 2018

\begin{abstract}
The objective of this paper was to systematically evaluate the potential preventive and therapeutic effects of exercise in attenuating stress-induced memory impairment. A systematic review was employed, searching PubMed, PsychInfo, Sports Discus and Google Scholar databases. For eligibility, studies had to be published in English, employ an experimental design, have the acute or chronic bout of exercise occur prior to, during or after the stressor, implement a psychophysiological stressor, and have an assessment of memory function occurring after the stressor. In total, 23 studies were evaluated, all of which were conducted among animal models. All 23 studies employed a chronic exercise protocol and a chronic stress protocol. Eight studies evaluated a preventive model, three employed a concurrent model, ten studies employed a therapeutic model, and two studies evaluated both a preventive and therapeutic model within the same study. Among the eight studies employing a preventive model, all eight demonstrated that the stress regimen impaired memory function. In all eight of these studies, when exercise occurred prior to the stressor, exercise attenuated the stress-induced memory impairment effect. Among the ten studies employing a therapeutic model, one study showed that the stress protocol enhanced memory function, one showed that the stress protocol did not influence memory, and eight demonstrated that the stress regimen impaired memory function. Among the eight studies showing that the stress protocol impaired memory function, all eight studies demonstrated that exercise, after the stressor, attenuated stress-induced memory impairment. Within animal models, chronic stress is associated with memory impairment and chronic exercise has both a preventive and therapeutic effect in attenuating stressinduced memory impairment. Additional experimental work in human studies is needed. Such work should also examine acute exercise and stress protocols.
\end{abstract}

Keywords Cognition · Exercise $\cdot$ Memory $\cdot$ Physical activity $\cdot$ Preventive $\cdot$ Psychological $\cdot$ Psychophysiological $\cdot$ Rescue . Stress $\cdot$ Therapeutic $\cdot$ Treatment

\section{Introduction}

The prophylactic and treatment effects of exercise on various chronic diseases is well established [1]. Additionally, exercise can also help to prevent a host of cardiometabolicrelated conditions (e.g., diabetes, early mortality) [2, 3]. Emerging work also demonstrates that, exercise, prior to a psychophysiological stressor [herein focused on toxic stress (not eustress)] [4], can mitigate the negative effects of the

Paul D. Loprinzi

pdloprin@olemiss.edu

1 Exercise \& Memory Laboratory, Department of Health, Exercise Science and Recreation Management, The University of Mississippi, 229 Turner Center, University, MS 38677, USA stressor. For example, we showed that acute exercise, occurring immediately before viewing emotionally charged, negatively valenced images, helped facilitate emotional regulation [5]. This "exercise preventive paradigm" has also been corroborated with other emotional regulation studies $[6,7]$.

This exercise preventive paradigm effect may also have implications in memory function. Psychophysiological stressors, such as forced social participation in a verbal presentation task, may have a negative effect on memory function [8-14]. Notably, this stress-memory relationship is thought to follow an inverted U-shaped relationship [15]. See Fig. 1 (and the Discussion section) for a schematic on the potential underlying mechanisms through which stress (both acute and chronic) may influence memory function. To our knowledge, however, there are no published reviews comprehensively evaluating the literature regarding the 


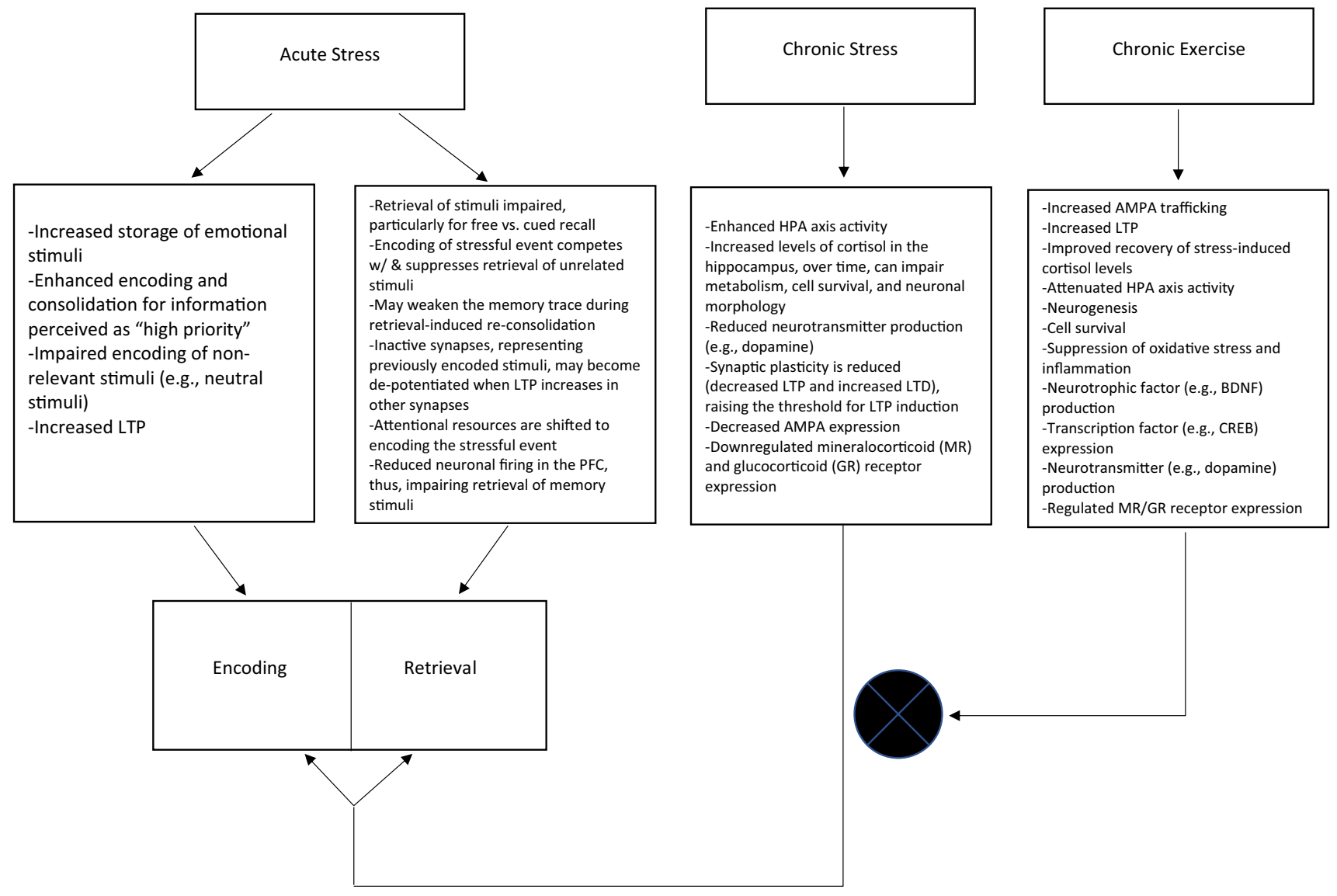

Fig. 1 Schematic indicating potential mechanisms through which acute stress may enhance memory, detrimental effects of chronic stress on memory, and how exercise may attenuate stress-induced

potential protective and/or therapeutic effects of exercise on mitigating stress-induced memory impairment [16], which was the purpose of this brief systematic review. The plausibility for exercise to attenuate stress-induced memory impairment is also shown in Fig. 1 (and further addressed in the Discussion section).

\section{Methods}

Studies were identified using electronic databases, including PubMed, PsychInfo, Sports Discus, and Google Scholar. We employed the computerized searches on April 25, 2018, identifying articles published prior to this date (no restriction was placed on how far back the study was published). The search terms included exercise, physical activity, stress, psychophysiological, rescue, preventive, treatment, therapeutic, psychological, memory, and cognition. To be eligible for inclusion in this systematic review, studies had to be published in English, employ an experimental design (crosssectional designs on this topic were not eligible) [17], have memory impairment. The circular crossed symbol denotes that chronic exercise attenuates the chronic stress mechanisms

the acute or chronic bout of exercise occur prior to, during, or after the stressor, implement a toxic psychophysiological stressor (pharmaceutical agent or ischemia-induction were not eligible [18-22], mild forms of the stressor were not eligible [23], and evaluating individuals without experimentally manipulating stress were not eligible) [24], and have an assessment of memory function that occurred after the stressor. To provide a comprehensive assessment on this topic, we applied no restriction on whether the study was conducted in humans or animal models. In total, 24 studies met our criteria. However, two appeared to be duplicate studies $[25,26]$, and thus, one was removed. As noted in Table 1, 23 studies were evaluated herein.

\section{Results}

Table 1 displays the extraction table for the evaluated studies. All were experimental studies conducted in an animal model. The stress protocol across the studies varied, including maternal separation, loud noise exposure, 
The Journal of Physiological Sciences (2019) 69:1-12

3

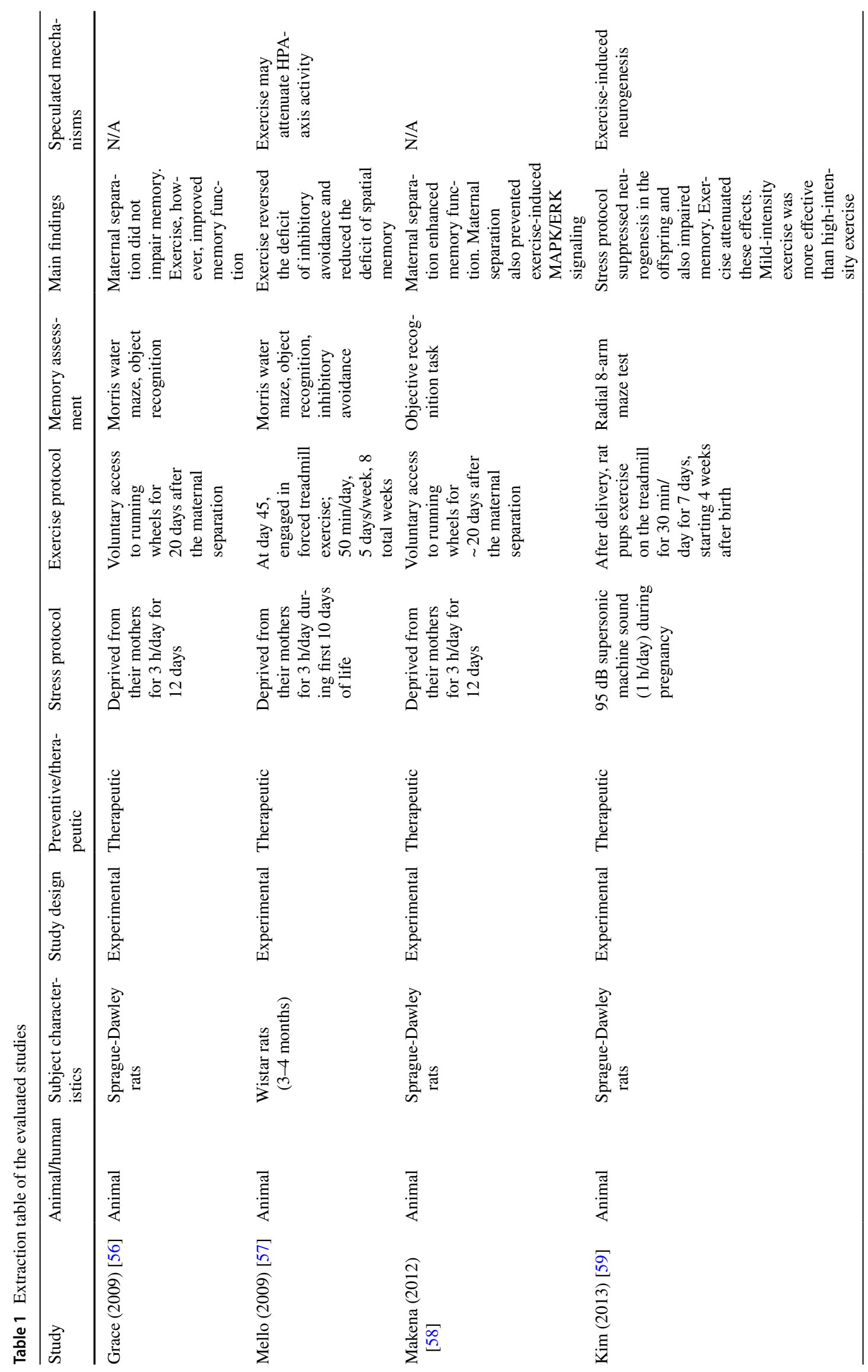

Springer 


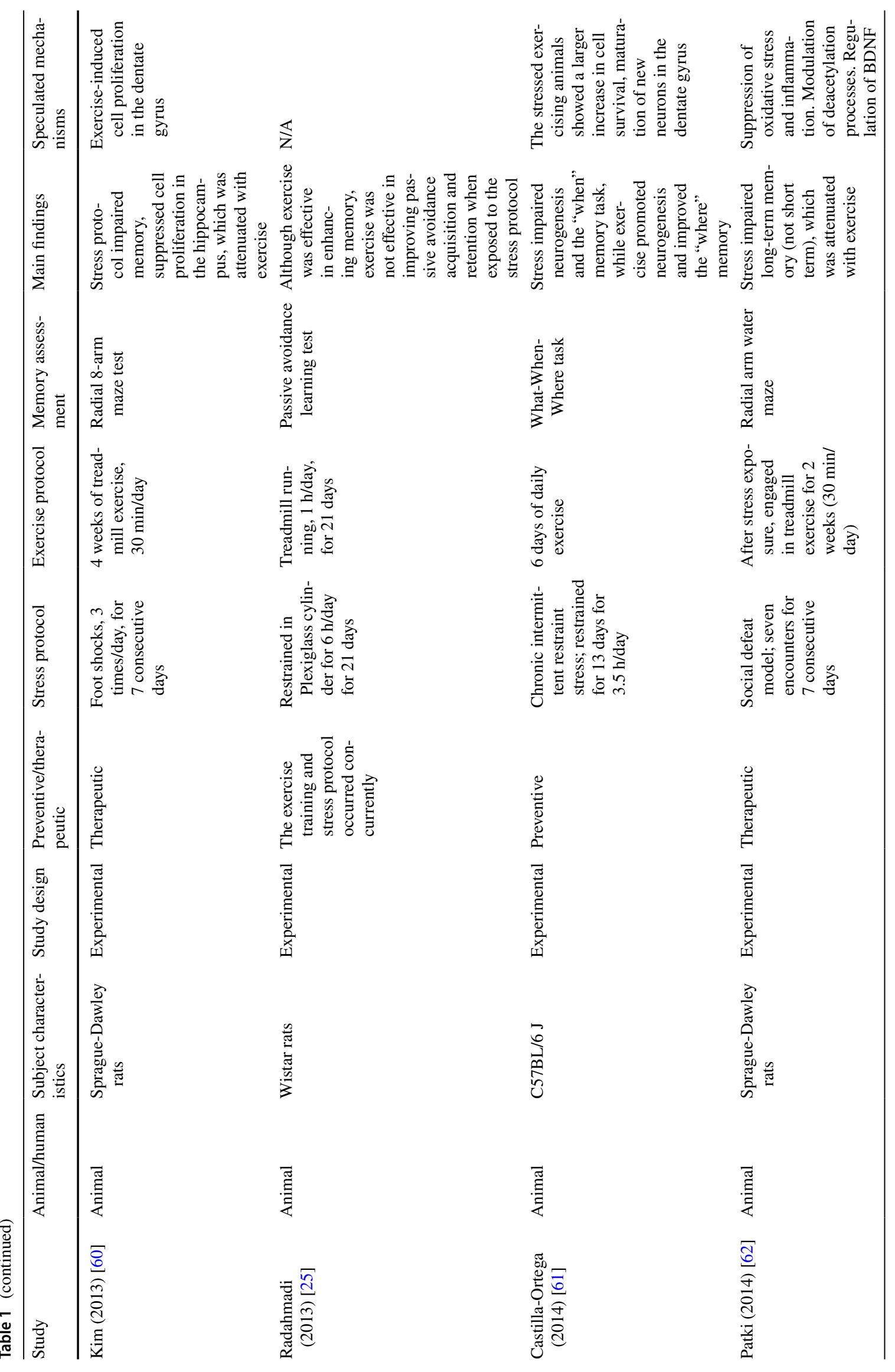




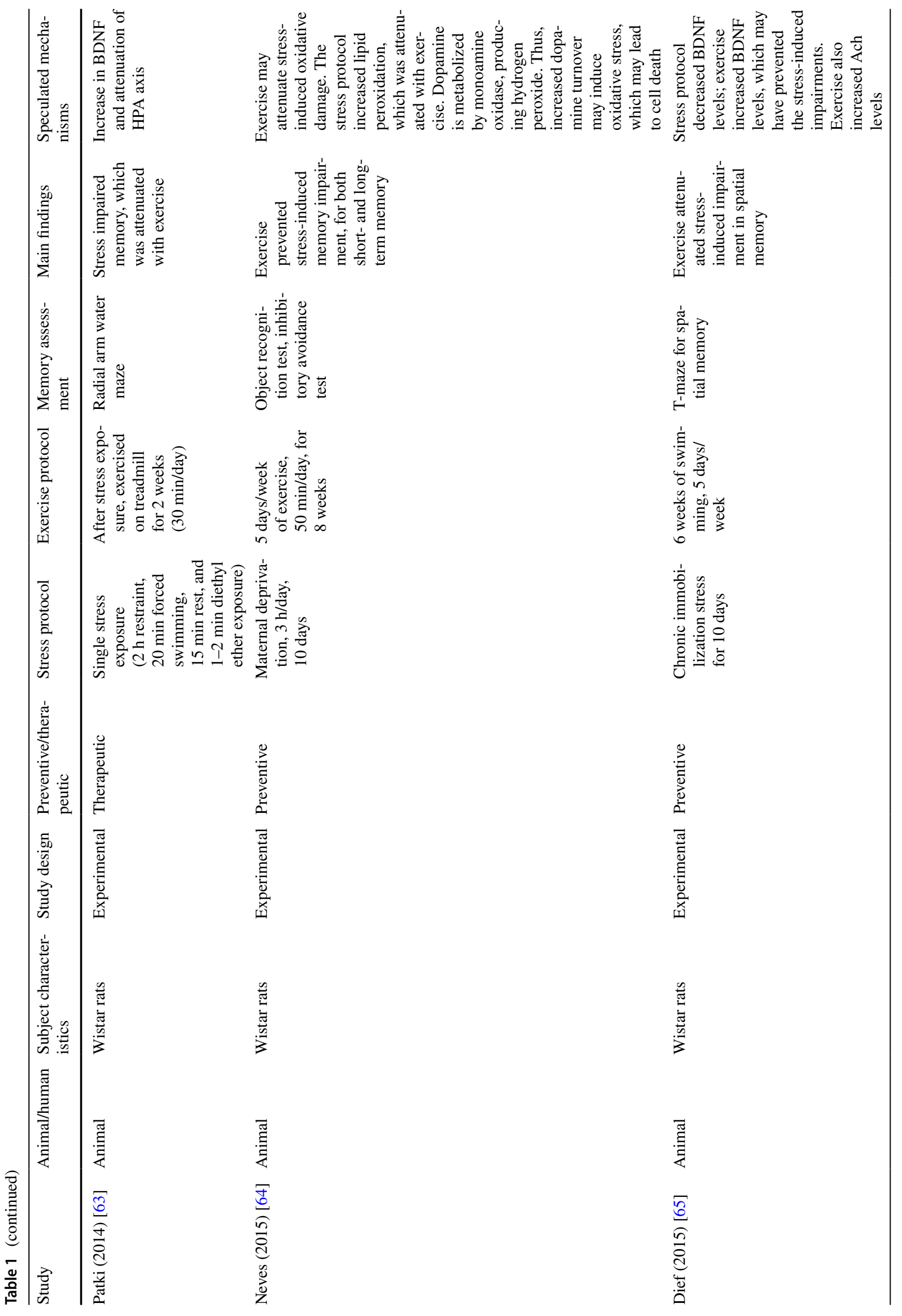




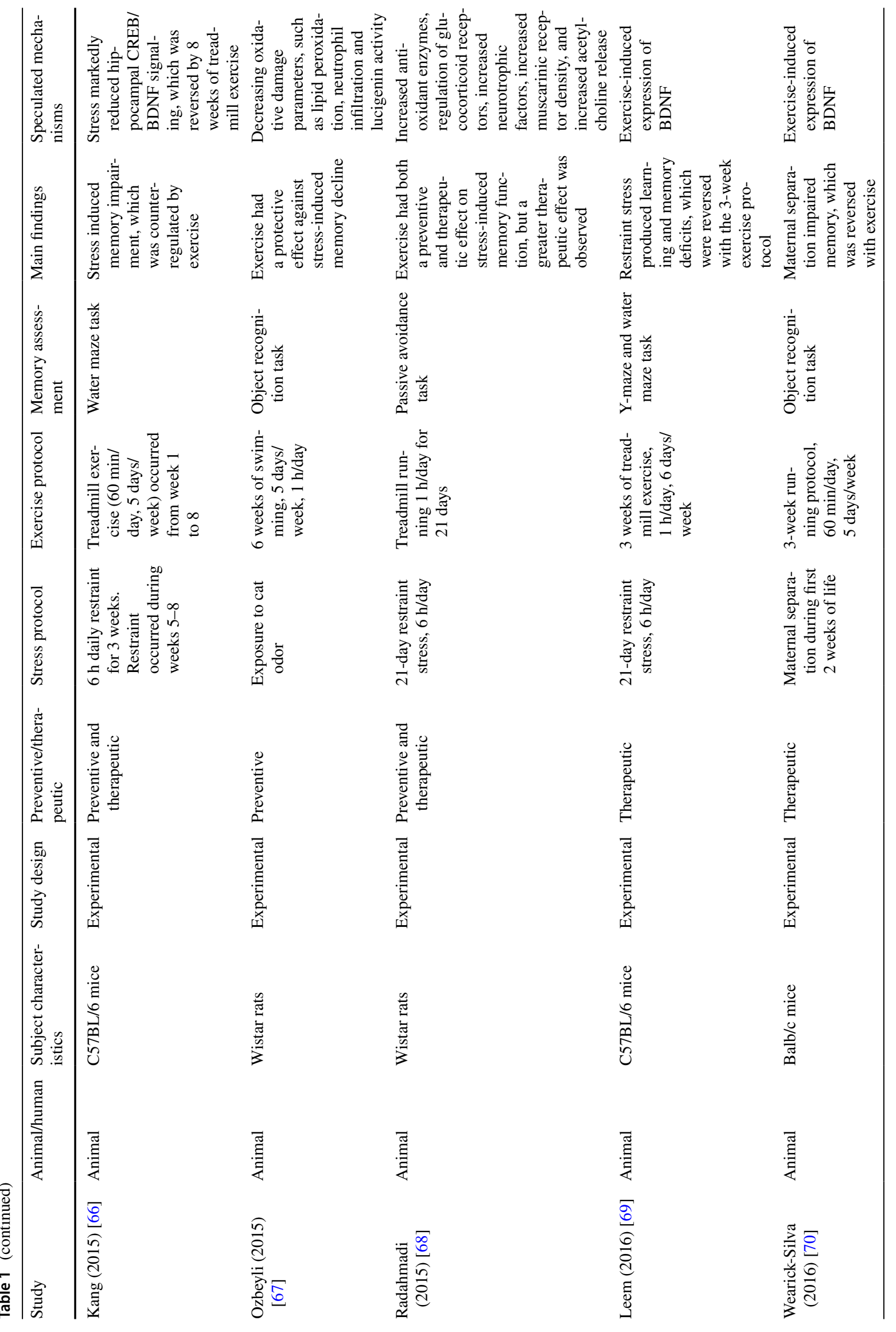




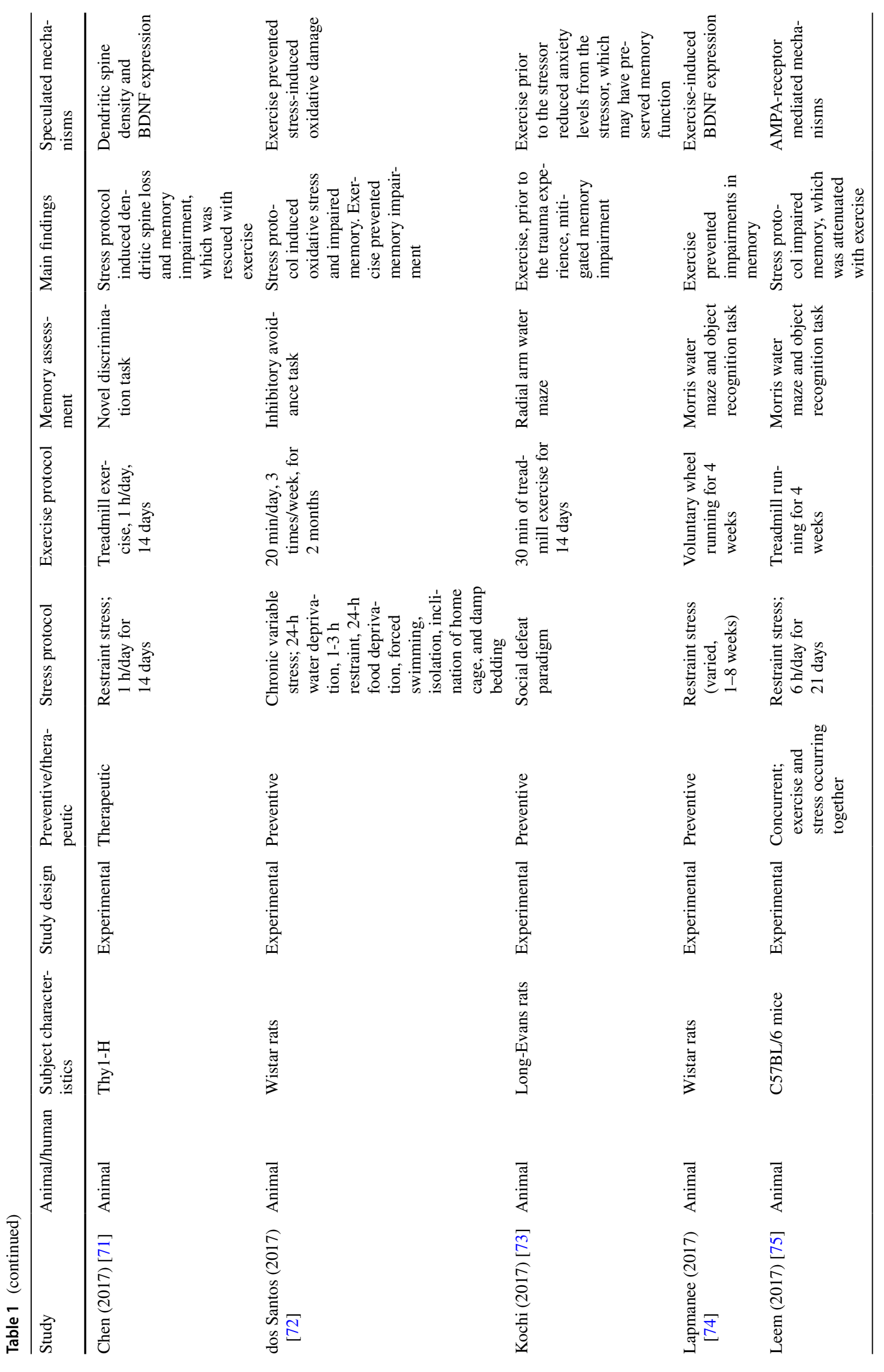




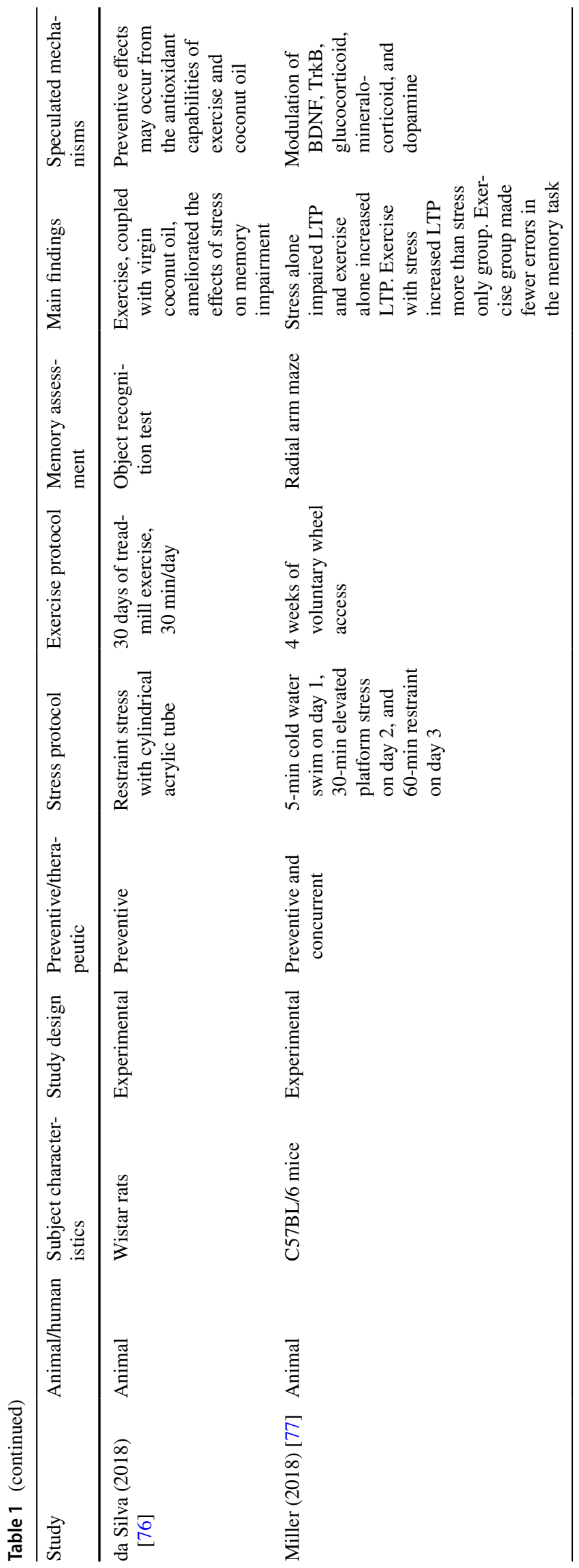

immobilization/restraint, social defeat/competition and exposure to cat odor. All studies, except one (single session acute stress protocol), employed a chronic stress protocol (e.g., multiple repeated exposures over 1-2 weeks). All 23 studies employed a chronic exercise protocol (e.g., daily exercise from 2 to 8 weeks; either forced treadmill exercise or voluntary wheel access). Among the 23 studies, the commonly assessed memory tasks included the Morris water maze, object recognition test, or inhibitory avoidance task.

Eight studies evaluated a preventive model (i.e., exercise occurring prior to stress-induction), three employed a concurrent model (exercise bout occurred during or around the same time as the stress protocol), ten studies employed a therapeutic model (i.e., exercise occurring after stressinduction), and two studies evaluated both a preventive and therapeutic model within the same study.

Among the eight studies employing a preventive model, all eight demonstrated that the stress regimen impaired memory function. In all eight of these studies, when exercise occurred prior to the stressor, exercise attenuated the stress-induced memory impairment effect.

Among the ten studies employing a therapeutic model, one study showed that the stress protocol enhanced memory function, one showed that the stress protocol did not influence memory, and eight demonstrated that the stress regimen impaired memory function. Among the eight studies showing that the stress protocol impaired memory function, all eight studies demonstrated that exercise, after the stressor, attenuated stress-induced memory impairment.

Among the three concurrent models, and the two studies that evaluated both preventive and therapeutic effects, all showed that the stress protocol impaired memory function. Among the three concurrent models, two demonstrated a beneficial effect of exercise in mitigating stress-induced memory impairment. Among the two studies employing both a preventive and therapeutic model, both demonstrated attenuation effects of exercise on stress-induced memory impairment.

\section{Discussion}

The objective of this systematic review was to evaluate the potential preventive and therapeutic effects of exercise in attenuating stress-induced memory impairment. There was consistent evidence that chronic exercise had both a preventive and therapeutic effect in mitigating chronic stressinduced memory impairment. The narrative that follows will discuss these mechanistic pathways, as displayed in Fig. 1. For additional discussion on these mechanisms, the reader is referred elsewhere $[16,27,28]$. 


\section{Acute stress and memory}

Acute moderate levels of stress may enhance memory, particularly emotional-based information (vs neutral stimuli). Specifically, enhanced encoding and consolidation of stimuli is more likely to occur for information perceived as "high priority" [29-33]. The stressor, occurring prior to the memory task, may help to augment attentional resources (via, for example, the prefrontal and parietal structures) to the memory stimuli and, in turn, enhance encoding of the information $[34,35]$. In addition to psychological stress, emerging work also suggests that exercise-induced physiological arousal may help to subserve stress-related memory function (emotional memory) [36]. Additional work is needed to determine whether there is an additive effect of exercise and acute stress on memory function.

Additionally, the stressor (including exercise) [36], occurring before, or shortly after, the memory task can help to facilitate the consolidation of the memory trace. For example, cortisol crosses the blood-brain barrier and binds to mineralocorticoid or glucocorticoid receptors. After which, PKA activation may help to facilitate exocytosis of AMPA receptors (and activation of NMDA receptors) [37], subserving hippocampal LTP [38]. Acute stress may also induce levels of epinephrine, activating the vagus nerve and, in turn, facilitating LTP via neurotransmitter (e.g., norepinephrine, dopamine, serotonin, and acetylcholine) production to the hippocampus [39-42]. To illustrate, the vagus nerve may stimulate the production of norepinephrine from the locus coeruleus, which then binds to adrenergic receptors, ultimately facilitating a cascade of intracellular signaling to induce synaptic plasticity [43]. Moreover, cortisol may augment endocannabinoid levels, binding to CB1 receptors in GABA interneurons and, ultimately, inhibiting GABA neurotransmitter levels [44]. This, in turn, may help to preserve memory, as GABA inhibition may help facilitate LTP [45] and GABA receptor activation may impair memory [46].

Although acute stress, occurring before encoding or during the early stages of consolidation, can facilitate encoding and consolidation of the prioritized stimuli, it can have the opposite effect on non-prioritized stimuli. The encoding of the stressful event may compete with the encoding of non-relevant or non-prioritized stimuli. Further, if the stressor occurs around the period of retrieving a memory, this memory retrieval process can be impaired, as attentional resources are shifted away from retrieval processes to encoding the stressful event. Additionally, inactive synapses, representing previously encoded stimuli, may become de-potentiated when LTP increases in other synapses [28]. Moreover, during the stressor, reduced neuronal firing may occur in the prefrontal cortex, which may impair memory retrieval since the prefrontal cortex plays an important role in such retrieval processes [47]. It would be worthwhile to investigate whether acute exercise can attenuate these effects by, for example, attenuating the stress response and facilitating emotional regulation [5].

Taken together, acute moderate levels of stress may help to facilitate encoding and consolidation of prioritized stimuli (particularly emotional stimuli), whereas extreme acute stressors may detrimentally influence retrieval of memories when the stressor occurs around the time of retrieving an unrelated memory. Notably, and as discussed next, chronic elevations in cortisol, lasting more than a few hours, can impair memory function (inducing LTD) [38].

\section{Chronic stress and memory}

Chronic stress may detrimentally influence stress through various mechanisms, including enhanced HPA axis activity. Over time, this may impair cell survival and neuronal morphology (e.g., loss of spines, shrinkage of dendrites) [4]. Regarding cell survival, astrocytes, which support the survival of neurons, possess glucocorticoid receptors and are significantly affected by chronic psychosocial stress [48]. Considering neuronal morphology, reduced synaptic firing, via LTD for example, causes actin loss and dendritic spine shrinkage [49]. Further, chronic stress may reduce BDNF levels [50], which play an important role in facilitating signaling pathways (e.g., RAC1) that stabilize dendritic spines [51]. Additionally, chronic stress may reduce neurotransmitter levels (e.g., dopamine) [52], decrease AMPA receptor expression [53], and downregulate mineralocorticoid and glucocorticoid receptor expression [54]. This downregulation and desensitization of these receptors may prevent activation of some of the above-mentioned cellular pathways (e.g., PKA) that may facilitate LTP. Further, chronic stress may inhibit neurogenesis, and ultimately, hippocampal volume loss, via, for example, apoptosis of progenitor cells to cell cycle arrest [49].

\section{Exercise mitigates negative effects of chronic stress}

Exercise may attenuate the memory-related consequences of chronic stress via various pathways. Ultimately, exercise may help to facilitate LTP through induced neuronal excitability, via stimulation of the vagus nerve as well as muscle afferent nerve fibers [55], which have direct projections to the brainstem and, ultimately, the hippocampus. Further, exercise-induced alterations in hormones (e.g., epinephrine and cortisol) can also influence neuronal excitability. Facilitating these effects, exercise has been shown to enhance neurotrophic factors (e.g., BDNF), induce transcription factors (e.g., CREB) expression, and increase AMPA trafficking [55]. Exercise may also help attenuate chronic stressinduced memory impairment via attenuation of HPA axis activity, suppress oxidative stress, facilitate neurogenesis, 
and regulate mineralocorticoid and glucocorticoid receptor expression [27].

\section{Conclusion}

This review demonstrates that, within animal models, chronic stress is associated with memory impairment and chronic exercise has both a preventive and therapeutic effect in attenuating stress-induced memory impairment. Given the paucity of work among human studies, future work on this topic among humans should investigate, specifically, whether exercise has a preventive and therapeutic effect in mitigating memory impairment caused from psychophysiological stress. Such work should also consider models that evaluate acute exercise and acute stress protocols. Further, work should also evaluate varying parameters of exercise, such as the intensity, duration, and type of exercise, as variations of these dimensions may have a unique influence on potentially attenuating stress-induced memory impairment.

\section{Compliance with ethical standards}

Conflict of interest Author PL declares no conflict of interest. Author EF declares no conflict of interest.

Research involving human participants All procedures performed in studies involving human participants were in accordance with the ethical standards of the institutional and/or national research committee and with the 1964 Helsinki Declaration and its later amendments or comparable ethical standards.

Informed consent No consent was needed as this is a review paper.

\section{References}

1. Kujala UM (2009) Evidence on the effects of exercise therapy in the treatment of chronic disease. Br J Sports Med 43(8):550-555. https://doi.org/10.1136/bjsm.2009.059808

2. Warburton DE, Nicol CW, Bredin SS (2006) Health benefits of physical activity: the evidence. CMAJ 174(6):801-809. https:// doi.org/10.1503/cmaj.051351

3. Warburton DER, Bredin SSD (2017) Health benefits of physical activity: a systematic review of current systematic reviews. Curr Opin Cardiol 32(5):541-556. https://doi.org/10.1097/HCO.00000 00000000437

4. McEwen BS (2017) Neurobiological and systemic effects of chronic stress. Chronic Stress (Thousand Oaks). https://doi. org/10.1177/2470547017692328

5. Edwards MK, Rhodes RE, Loprinzi PD (2017) A randomized control intervention investigating the effects of acute exercise on emotional regulation. Am J Health Behav 41(5):534-543. https:// doi.org/10.5993/AJHB.41.5.2

6. Bernstein EE, McNally RJ (2017) Acute aerobic exercise helps overcome emotion regulation deficits. Cogn Emot 31(4):834-843. https://doi.org/10.1080/02699931.2016.1168284
7. Bernstein EE, McNally RJ (2017) Acute aerobic exercise hastens emotional recovery from a subsequent stressor. Health Psychol 36(6):560-567. https://doi.org/10.1037/hea0000482

8. de Quervain DJ, Henke K, Aerni A et al (2003) Glucocorticoidinduced impairment of declarative memory retrieval is associated with reduced blood flow in the medial temporal lobe. Eur J Neurosci 17(6):1296-1302

9. de Quervain DJ, Roozendaal B, Nitsch RM et al (2000) Acute cortisone administration impairs retrieval of long-term declarative memory in humans. Nat Neurosci 3(4):313-314. https://doi. org/10.1038/73873

10. Het S, Ramlow G, Wolf OT (2005) A meta-analytic review of the effects of acute cortisol administration on human memory. Psychoneuroendocrinology 30(8):771-784. https://doi.org/10.1016/j. psyneuen.2005.03.005

11. de Quervain DJ, Roozendaal B, McGaugh JL (1998) Stress and glucocorticoids impair retrieval of long-term spatial memory. Nature 394(6695):787-790. https://doi.org/10.1038/29542

12. Diamond DM, Park CR, Heman KL et al (1999) Exposing rats to a predator impairs spatial working memory in the radial arm water maze. Hippocampus 9(5):542-552. https://doi.org/10.1002/ (SICI)1098-1063(1999)9:5\%3c542:AID-HIPO8\%3e3.0.CO;2-N

13. Park CR, Zoladz PR, Conrad CD et al (2008) Acute predator stress impairs the consolidation and retrieval of hippocampus-dependent memory in male and female rats. Learn Mem 15(4):271-280. https://doi.org/10.1101/lm.721108

14. Guez J, Saar-Ashkenazy R, Keha E et al (2016) The effect of Trier social stress test (TSST) on item and associative recognition of words and pictures in healthy participants. Front Psychol 7:507. https://doi.org/10.3389/fpsyg.2016.00507

15. Salehi B, Cordero MI, Sandi C (2010) Learning under stress: the inverted-U-shape function revisited. Learn Mem 17(10):522-530. https://doi.org/10.1101/lm.1914110

16. Leem YH (2017) The potential role of exercise in chronic stressrelated changes in AMPA receptor phenotype underlying synaptic plasticity. J Exerc Nutr Biochem 21(4):11-15. https://doi. org/10.20463/jenb.2017.0037

17. Head D, Singh T, Bugg JM (2012) The moderating role of exercise on stress-related effects on the hippocampus and memory in later adulthood. Neuropsychology 26(2):133-143. https://doi. org/10.1037/a0027108

18. Schimidt HL, Vieira A, Altermann C et al (2014) Memory deficits and oxidative stress in cerebral ischemia-reperfusion: neuroprotective role of physical exercise and green tea supplementation. Neurobiol Learn Mem 114:242-250. https://doi.org/10.1016/j. nlm.2014.07.005

19. Shih PC, Yang YR, Wang RY (2013) Effects of exercise intensity on spatial memory performance and hippocampal synaptic plasticity in transient brain ischemic rats. PLoS One 8(10):e78163. https ://doi.org/10.1371/journal.pone.0078163

20. Wang Q, Xu Z, Tang J et al (2013) Voluntary exercise counteracts Abeta25-35-induced memory impairment in mice. Behav Brain Res 256:618-625. https://doi.org/10.1016/j.bbr.2013.09.024

21. Cechetti F, Worm PV, Elsner VR et al (2012) Forced treadmill exercise prevents oxidative stress and memory deficits following chronic cerebral hypoperfusion in the rat. Neurobiol Learn Mem 97(1):90-96. https://doi.org/10.1016/j.nlm.2011.09.008

22. Gozal D, Nair D, Goldbart AD (2010) Physical activity attenuates intermittent hypoxia-induced spatial learning deficits and oxidative stress. Am J Respir Crit Care Med 182(1):104-112. https:// doi.org/10.1164/rccm.201001-0108OC

23. Adlard PA, Engesser-Cesar C, Cotman CW (2011) Mild stress facilitates learning and exercise improves retention in aged mice. Exp Gerontol 46(1):53-59. https://doi.org/10.1016/j.exger .2010 .10 .001 
24. Zlomuzica A, Woud ML, Machulska A et al (2018) Deficits in episodic memory and mental time travel in patients with posttraumatic stress disorder. Prog Neuropsychopharmacol Biol Psychiatry 83:42-54. https://doi.org/10.1016/j.pnpbp.2017.12.014

25. Radahmadi M, Alaei H, Sharifi MR et al (2013) The effect of synchronized running activity with chronic stress on passive avoidance learning and body weight in rats. Int J Prev Med 4(4):430-437

26. Radahmadi M, Alaei H, Sharifi MR et al (2013) The effect of synchronized forced running with chronic stress on short, mid and long-term memory in rats. Asian J Sports Med 4(1):54-62

27. Chen C, Nakagawa S, An Y et al (2017) The exercise-glucocorticoid paradox: how exercise is beneficial to cognition, mood, and the brain while increasing glucocorticoid levels. Front Neuroendocrinol 44:83-102. https://doi.org/10.1016/j.yfrne.2016.12.001

28. Gagnon SA, Wagner AD (2016) Acute stress and episodic memory retrieval: neurobiological mechanisms and behavioral consequences. Ann N Y Acad Sci 1369(1):55-75. https://doi. org/10.1111/nyas.12996

29. Clewett DV, Huang R, Velasco R et al (2018) Locus coeruleus activity strengthens prioritized memories under arousal. J Neurosci 38(6):1558-1574. https://doi.org/10.1523/JNEUR OSCI.2097-17.2017

30. Clewett D, Sakaki M, Huang R et al (2017) Arousal amplifies biased competition between high and low priority memories more in women than in men: the role of elevated noradrenergic activity. Psychoneuroendocrinology 80:80-91. https://doi.org/10.1016/j. psyneuen.2017.02.022

31. Lee TH, Itti L, Mather M (2012) Evidence for arousal-biased competition in perceptual learning. Front Psychol 3:241. https://doi. org/10.3389/fpsyg.2012.00241

32. Lee TH, Sakaki M, Cheng R et al (2014) Emotional arousal amplifies the effects of biased competition in the brain. Soc Cogn Affect Neurosci 9(12):2067-2077. https://doi.org/10.1093/scan/nsu015

33. Mather M, Sutherland MR (2011) Arousal-biased competition in perception and memory. Perspect Psychol Sci 6(2):114-133. https ://doi.org/10.1177/1745691611400234

34. Dehaene S, Kerszberg M, Changeux JP (1998) A neuronal model of a global workspace in effortful cognitive tasks. Proc Natl Acad Sci USA 95(24):14529-14534

35. Daffner KR, Scinto LF, Weitzman AM et al (2003) Frontal and parietal components of a cerebral network mediating voluntary attention to novel events. J Cogn Neurosci 15(2):294-313

36. Loprinzi PD, Frith E, Edwards MK (2018) Exercise and emotional memory: a systematic review. J Cogn Enhanc. https://doi. org/10.1007/s41465-018-0086-Z

37. Whitehead G, Jo J, Hogg EL et al (2013) Acute stress causes rapid synaptic insertion of $\mathrm{Ca}^{2+}$-permeable AMPA receptors to facilitate long-term potentiation in the hippocampus. Brain $136(\mathrm{Pt}$ 12):3753-3765. https://doi.org/10.1093/brain/awt293

38. Joels M, Krugers HJ (2007) LTP after stress: up or down? Neural Plast 2007:93202. https://doi.org/10.1155/2007/93202

39. Marzo A, Bai J, Otani S (2009) Neuroplasticity regulation by noradrenaline in mammalian brain. Curr Neuropharmacol 7(4):286-295. https://doi.org/10.2174/157015909790031193

40. Morcom AM, Bullmore ET, Huppert FA et al (2010) Memory encoding and dopamine in the aging brain: a psychopharmacological neuroimaging study. Cereb Cortex 20(3):743-757. https ://doi.org/10.1093/cercor/bhp139

41. Mlinar B, Stocca G, Corradetti R (2015) Endogenous serotonin facilitates hippocampal long-term potentiation at CA3/CA1 synapses. J Neural Transm (Vienna) 122(2):177-185. https://doi. org/10.1007/s00702-014-1246-7
42. Hasselmo ME (2006) The role of acetylcholine in learning and memory. Curr Opin Neurobiol 16(6):710-715. https://doi. org/10.1016/j.conb.2006.09.002

43. Tully K, Bolshakov VY (2010) Emotional enhancement of memory: how norepinephrine enables synaptic plasticity. Mol Brain 3:15. https://doi.org/10.1186/1756-6606-3-15

44. Hill MN, McEwen BS (2010) Involvement of the endocannabinoid system in the neurobehavioural effects of stress and glucocorticoids. Prog Neuropsychopharmacol Biol Psychiatry 34(5):791797. https://doi.org/10.1016/j.pnpbp.2009.11.001

45. Chapman CA, Perez Y, Lacaille JC (1998) Effects of GABA(A) inhibition on the expression of long-term potentiation in CA1 pyramidal cells are dependent on tetanization parameters. Hippocampus 8(3):289-298. https://doi.org/10.1002/(SICI)10981063(1998)8:3\%3c289:AID-HIPO10\%3e3.0.CO;2-X

46. Krebs-Kraft DL, Wheeler MG, Parent MB (2007) The memoryimpairing effects of septal GABA receptor activation involve GABAergic septo-hippocampal projection neurons. Learn Mem 14(12):833-841. https://doi.org/10.1101/lm.809407

47. Preston AR, Eichenbaum H (2013) Interplay of hippocampus and prefrontal cortex in memory. Curr Biol 23(17):R764-773. https:// doi.org/10.1016/j.cub.2013.05.041

48. Czeh B, Simon M, Schmelting B et al (2006) Astroglial plasticity in the hippocampus is affected by chronic psychosocial stress and concomitant fluoxetine treatment. Neuropsychopharmacology 31(8):1616-1626. https://doi.org/10.1038/sj.npp.1300982

49. Krugers HJ, Lucassen PJ, Karst H et al (2010) Chronic stress effects on hippocampal structure and synaptic function: relevance for depression and normalization by anti-glucocorticoid treatment. Front Synaptic Neurosci 2:24. https://doi.org/10.3389/fnsyn .2010 .00024

50. Murakami S, Imbe H, Morikawa Y et al (2005) Chronic stress, as well as acute stress, reduces BDNF mRNA expression in the rat hippocampus but less robustly. Neurosci Res 53(2):129-139. https ://doi.org/10.1016/j.neures.2005.06.008

51. Koleske AJ (2013) Molecular mechanisms of dendrite stability. Nat Rev Neurosci 14(8):536-550. https://doi.org/10.1038/nrn34 86

52. Alghasham A, Rasheed N (2014) Stress-mediated modulations in dopaminergic system and their subsequent impact on behavioral and oxidative alterations: an update. Pharm Biol 52:368-377

53. Kallarackal AJ, Kvarta MD, Cammarata E et al (2013) Chronic stress induces a selective decrease in AMPA receptor-mediated synaptic excitation at hippocampal temporoammonic-CA1 synapses. J Neurosci 33(40):15669-15674. https://doi.org/10.1523/ JNEUROSCI.2588-13.2013

54. Meyer U, van Kampen M, Isovich E et al (2001) Chronic psychosocial stress regulates the expression of both GR and MR mRNA in the hippocampal formation of tree shrews. Hippocampus 11(3):329-336. https://doi.org/10.1002/hipo.1047

55. Loprinzi PD, Edwards MK, Frith E (2017) Potential avenues for exercise to activate episodic memory-related pathways: a narrative review. Eur J Neurosci 46(5):2067-2077. https://doi.org/10.1111/ ejn. 13644

56. Grace L, Hescham S, Kellaway LA et al (2009) Effect of exercise on learning and memory in a rat model of developmental stress. Metab Brain Dis 24(4):643-657. https://doi.org/10.1007/s1101 1-009-9162-5

57. Mello PB, Benetti F, Cammarota M et al (2009) Physical exercise can reverse the deficit in fear memory induced by maternal deprivation. Neurobiol Learn Mem 92(3):364-369. https://doi. org/10.1016/j.nlm.2009.04.004

58. Makena N, Bugarith K, Russell VA (2012) Maternal separation enhances object location memory and prevents exerciseinduced MAPK/ERK signalling in adult Sprague-Dawley rats. 
Metab Brain Dis 27(3):377-385. https://doi.org/10.1007/s1101 1-012-9298-6

59. Kim TW, Shin MS, Park JK et al (2013) Treadmill exercise alleviates prenatal noise stress-induced impairment of spatial learning ability through enhancing hippocampal neurogenesis in rat pups. J Exerc Rehabil 9(5):451-456. https://doi.org/10.12965/jer.13006 4

60. Kim BK, Seo JH (2013) Treadmill exercise alleviates posttraumatic stress disorder-induced impairment of spatial learning memory in rats. J Exerc Rehabil 9(4):413-419. https://doi. org/10.12965/jer.130058

61. Castilla-Ortega E, Rosell-Valle C, Pedraza C et al (2014) Voluntary exercise followed by chronic stress strikingly increases mature adult-born hippocampal neurons and prevents stressinduced deficits in 'what-when-where' memory. Neurobiol Learn Mem 109:62-73. https://doi.org/10.1016/j.nlm.2013.12.001

62. Patki G, Solanki N, Atrooz F et al (2014) Novel mechanistic insights into treadmill exercise based rescue of social defeatinduced anxiety-like behavior and memory impairment in rats. Physiol Behav 130:135-144. https://doi.org/10.1016/j.physb eh.2014.04.011

63. Patki G, Li L, Allam F et al (2014) Moderate treadmill exercise rescues anxiety and depression-like behavior as well as memory impairment in a rat model of posttraumatic stress disorder. Physiol Behav 130:47-53. https://doi.org/10.1016/j.physbeh.2014.03.016

64. Neves BH, Menezes J, Souza MA et al (2015) Physical exercise prevents short and long-term deficits on aversive and recognition memory and attenuates brain oxidative damage induced by maternal deprivation. Physiol Behav 152(Pt A):99-105. https:// doi.org/10.1016/j.physbeh.2015.09.019

65. Dief AE, Samy DM, Dowedar FI (2015) Impact of exercise and vitamin B1 intake on hippocampal brain-derived neurotrophic factor and spatial memory performance in a rat model of stress. J Nutr Sci Vitaminol 61:1-7

66. Kang JS (2015) Exercise copes with prolonged stress-induced impairment of spatial memory performance by endoplasmic reticulum stress. J Exerc Nutr Biochem 19(3):191-197. https:// doi.org/10.5717/jenb.2015.15080705

67. Ozbeyli D, Gokalp AG, Koral T et al (2015) Protective effect of exercise and sildenafil on acute stress and cognitive function. Physiol Behav 151:230-237. https://doi.org/10.1016/j.physb eh.2015.07.030

68. Radahmadi M, Alaei H, Sharifi MR et al (2015) Preventive and therapeutic effect of treadmill running on chronic stress-induced memory deficit in rats. J Bodyw Mov Ther 19(2):238-245. https ://doi.org/10.1016/j.jbmt.2014.04.007

69. Kim DM, Leem YH (2016) Chronic stress-induced memory deficits are reversed by regular exercise via AMPK-mediated BDNF induction. Neuroscience 324:271-285. https://doi.org/10.1016/j. neuroscience.2016.03.019

70. Wearick-Silva LE, Marshall P, Viola TW et al (2017) Running during adolescence rescues a maternal separation-induced memory impairment in female mice: potential role of differential exonspecific BDNF expression. Dev Psychobiol 59(2):268-274. https ://doi.org/10.1002/dev.21487

71. Chen K, Zhang L, Tan M et al (2017) Treadmill exercise suppressed stress-induced dendritic spine elimination in mouse barrel cortex and improved working memory via BDNF/TrkB pathway. Transl Psychiatry 7(3):e1069. https://doi.org/10.1038/tp.2017.41

72. Dos Santos TM, Kolling J, Siebert C et al (2017) Effects of previous physical exercise to chronic stress on long-term aversive memory and oxidative stress in amygdala and hippocampus of rats. Int J Dev Neurosci 56:58-67. https://doi.org/10.1016/j.ijdev neu.2016.12.003

73. Kochi C, Liu H, Zaidi S et al (2017) Prior treadmill exercise promotes resilience to vicarious trauma in rats. Prog Neuropsychopharmacol Biol Psychiatry 77:216-221. https://doi.org/10.1016/j. pnpbp.2017.04.018

74. Lapmanee S, Charoenphandhu J, Teerapornpuntakit J et al (2017) Agomelatine, venlafaxine, and running exercise effectively prevent anxiety- and depression-like behaviors and memory impairment in restraint stressed rats. PLoS One 12(11):e0187671. https ://doi.org/10.1371/journal.pone.0187671

75. Leem YH, Chang H (2017) Arc/Arg3.1 protein expression in dorsal hippocampal CA1, a candidate event as a biomarker for the effects of exercise on chronic stress-evoked behavioral abnormalities. J Exerc Nutr Biochem 21(4):45-51. https://doi.org/10.20463 /jenb.2017.0033

76. da Silva DC, Tavares MG, do Nascimento CKB et al (2018) Can coconut oil and treadmill exercise during the critical period of brain development ameliorate stress-related effects on anxiety-like behavior and episodic-like memory in young rats? Food Funct 9(3):1492-1499. https://doi.org/10.1039/c7fo01516j

77. Miller RM, Marriott D, Trotter J et al (2018) Running exercise mitigates the negative consequences of chronic stress on dorsal hippocampal long-term potentiation in male mice. Neurobiol Learn Mem 149:28-38. https://doi.org/10.1016/j.nlm.2018.01.008 